\title{
Less is more in treating myocardial infarction in younger patients: intracoronary optical coherence tomography insights
}

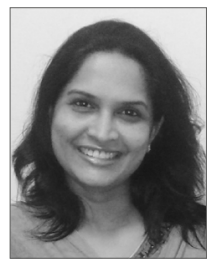

Suma M. Victor, DNB, FNB; Vijayakumar Subban, DM, FNB; Anandkumar Gopi, DNB, FNB;

Deva Preethi Rajaraman, BS; Ajit S. Mullasari*, DM, FRCP

The Madras Medical Mission, Chennai, India

This paper also includes supplementary data published online at: https://www.asiaintervention.org/doi/10.4244/AIJ-D-21-00015

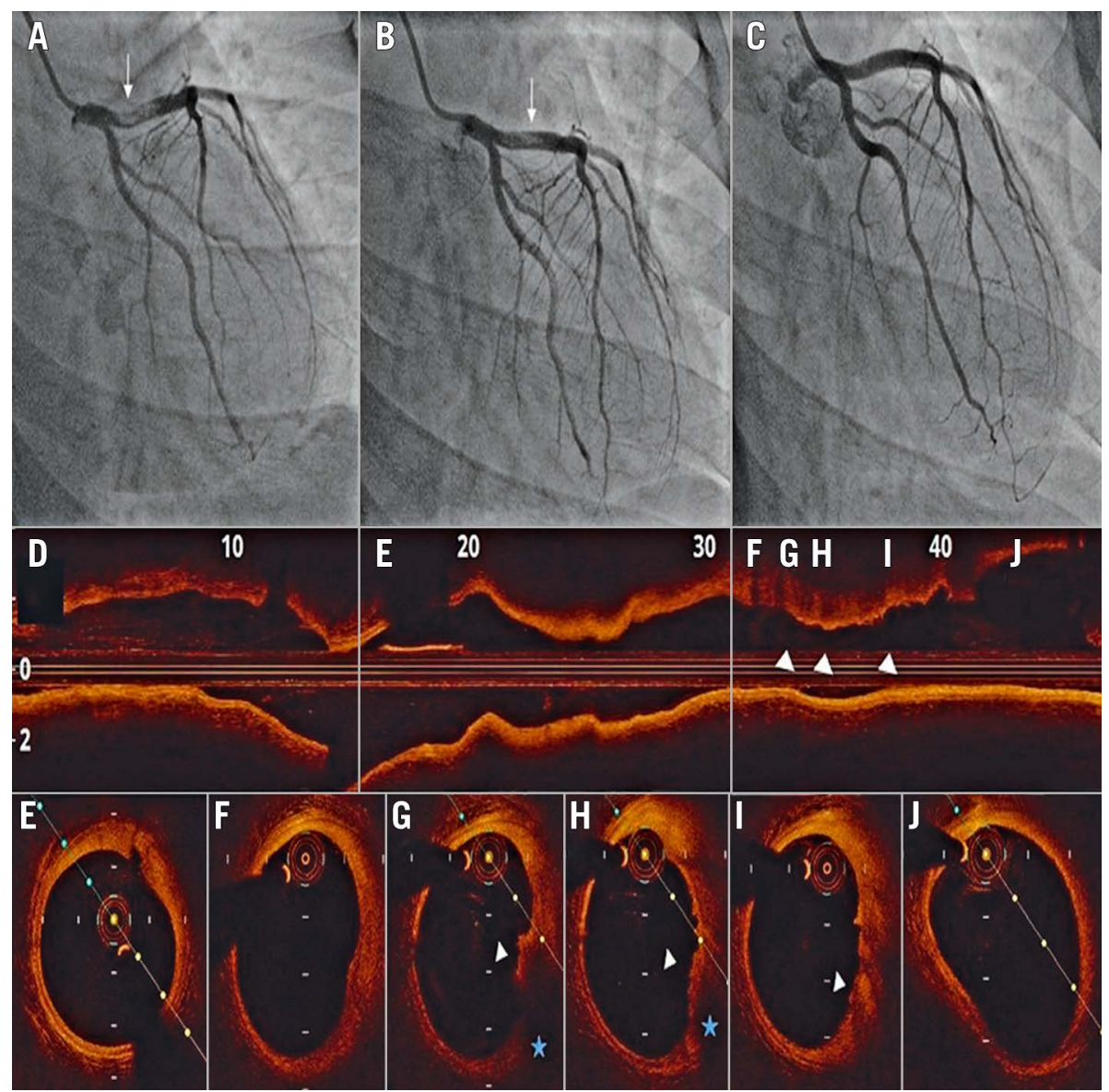

*Corresponding author: Institute of Cardiovascular Diseases, The Madras Medical Mission, 4A, Dr J J Nagar, Mogappair, Chennai, Tamil Nadu 600037,.India.E-mail: sulu_ajit57@yahoo.co.in 
Despite discouraging clinical trials, intracoronary thrombolysis may be an important and viable treatment option in select cases of ST-segment elevation myocardial infarction (STEMI), such as in younger patients with large thrombus burden for whom the underlying pathology is often plaque erosion. Here, we present an elegant demonstration of successful intracoronary lysis, which resulted in near resolution of thrombus.

A 29-year-old male presented with anterior STEMI with threehour chest pain. He was pre-treated with dual antiplatelets and statins followed by an emergency coronary catheterisation which showed a proximal left anterior descending coronary artery (LAD) lesion with large thrombus burden (Thrombolysis In Myocardial Infarction [TIMI] risk score of 4) and TIMI 2 flow (Panel A, Moving image 1). To avoid the inherent risk of distal or proximal embolisation with instrumentation of the artery, intracoronary lysis with $9 \mathrm{mg}$ of reteplase was given slowly through the diagnostic catheter. The post-lysis angiogram showed a significant reduction in thrombus burden with TIMI 3 flow (Panel B, Moving image 2) and complete ST-segment resolution (Supplementary Figure 1A, Supplementary Figure 1B). The patient was further treated with an eptifibatide infusion for 24 hours along with lowmolecular-weight heparin. A second angiogram after 48 hours showed complete thrombus resolution with no residual stenosis (Panel C, Moving image 3). Optical coherence tomography imaging demonstrated a small plaque burden with intact fibrous cap and minimal residual thrombus, suggestive of plaque erosion (Panel D-Panel J, Moving image 4). He was discharged the following day with dual antiplatelets and other recommended medication. Six months into follow-up, his treadmill test was negative and he remains symptom free.

\section{Conflict of interest statement}

The authors have no conflicts of interest to declare.

\section{Supplementary data}

Supplementary Figure 1. ECG at presentation and after intracoronary thrombolysis.

Moving image 1. Coronary angiogram showing a proximal LAD lesion with large thrombus burden.

Moving image 2. Reduction of thrombus burden post intracoronary lysis.

Moving image 3. Angiogram after 48 hours showing complete resolution of thrombus and no residual stenosis.

Moving image 4. OCT images.

The supplementary data are published online at:

https://www.asiaintervention.org/

doi/10.4244/AIJ-D-21-00015 


\section{Supplementary data}
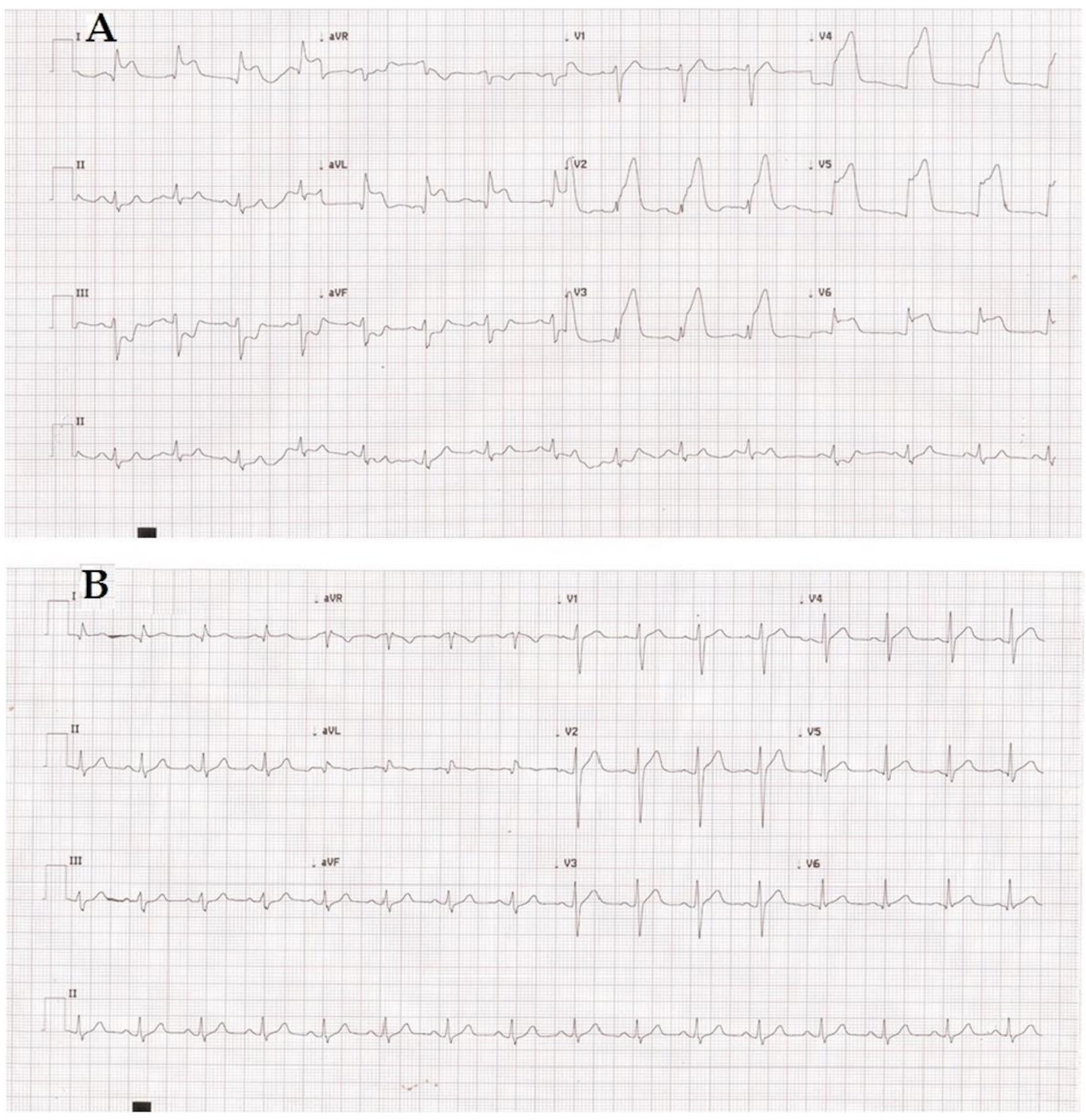

Supplementary Figure 1. ECG at presentation and after intracoronary thrombolysis.
A. ECG at presentation.
B. ECG after intracoronary lysis. 